\title{
Cooling Neutron Stars
}

\author{
Hakki Ögelman, Garching
}

(Max-Planck-Institut für Extraterrestrische Physik)

Historically, the first schemes for discovering neutron stars were based on the detection of the thermal radiation from a cooling star in the X-ray band. Since neutron stars are the remains of the central cores of massive stars that have consumed all possible internal energy sources, it was natural to assume that they would be cooling from the moment they were created. Following the advent of $\mathrm{X}$-ray astronomy in the early 1960 s with the discovery of the brightest galactic $X$-ray source Sco $X-1$ (Giacconi et al. 1962), detailed calculations of cooling rates and cooling curves started to appear in the scientific literature (for a review see Tsuruta 1986). The hope was to find neutron stars several hundred years old, with surface temperatures around several million degrees. Although the general idea was correct, this method of finding neutron stars turned out to be the difficult way.

It is ironic that only recently have we been able to detect thermal radiation from cooling neutron stars, after the development of sensitive focusing $X$-ray instruments - some 12 years after the discovery of neutron stars as pulsars (Hewish et al. 1968). In retrospect, it is obvious that during the collapse to a neutron star, the original magnetic field and the rotation rate of the progenitor star would be amplified by approximately the square of the ratio of the initial to final radius $\left(\cong 10^{10}\right)$, and that such objects should generate all kinds of electromagnetic fields and waves at the expense of their rotational energy. In the meantime, the strong $\mathrm{X}$-ray sources discovered five years before the pulsars have also been identified as neutron stars, accreting mass from close binary companions and converting the gravitational energy gained by the gas to $X$-rays emitted from the surface. Although this model was suggested back in 1967 by Shklovskii to explain Sco X-1, several years were to pass before the observations with the UHURU satellite showed the existence of pulsing $\mathrm{X}$-ray sources which were unmistakably interpreted as rotating and accreting neutron stars.

Despite the fact that the radiation emitted from cooling neutron stars has not been the best way to discover them, this information should contribute signi-

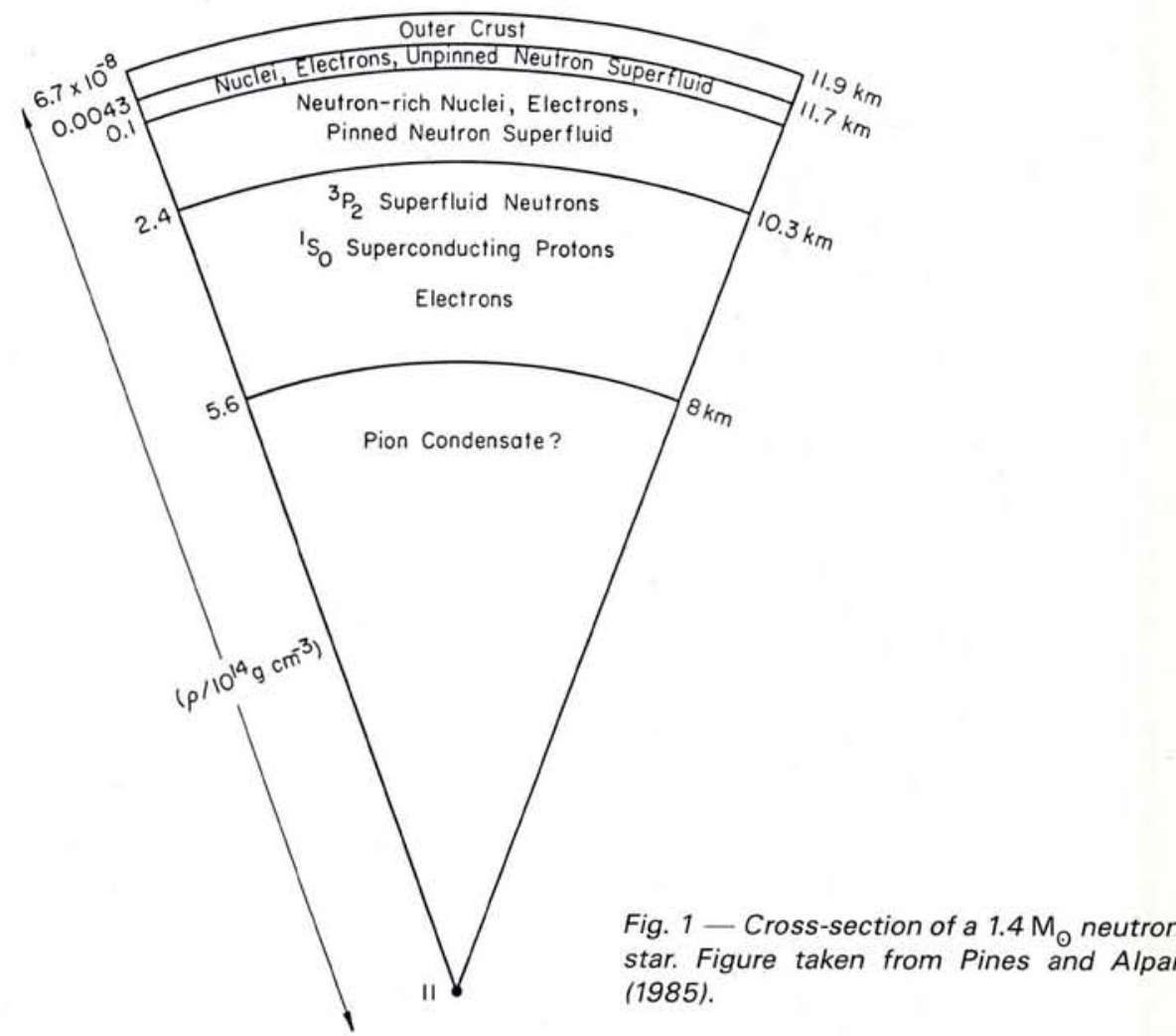

ficantly to our understanding of the internal structure of the neutron star. The structure is of topical interest not only for astrophysics, it also provides a unique opportunity to study the imperfectly understood hadron equation of state at nuclear $\left(\rho_{0} \cong 3 \times 10^{14} \mathrm{~g} / \mathrm{cm}^{3}\right)$ and higher densities (for reviews see Gordon, Baym and Pethick 1979; Pines 1987; Shapiro and Teukolsky 1983, Chapter 8). Let us start by examining the salient features of a 1.4 solar mass $\left(M_{0}\right.$ $=2 \times 10^{33} \mathrm{~g}$ ) "cold" neutron star with "stiff" equation of state, shown in Fig. 1 (from Pines and Alpar 1985). Going from outside to the centre, one encounters first an atmosphere a few meters thick, followed by an outer crust, a few hundred meters in depth and of density increasing from $7 \times 10^{6}$ to $4 \times 10^{11}$ $\mathrm{g} / \mathrm{cm}^{3}$. The outer crust consists of a solid array of nuclei and degenerate relativistic electrons. The next kilometer or so is called the inner crust; it starts with the density $4 \times 10^{11} \mathrm{~g} / \mathrm{cm}^{3}$, where the neutrons find it energetically easier to exist outside the nuclei and start dripping out, and extends to $\rho_{0}$, the nuclear density regime. The properties of this region which contains degenerate elec- trons, superfluid neutrons, and a lattice of neutron rich nuclei, is reasonably well understood. At deeper layers, going down 10 kilometers to the centre, where densities are in excess of $\rho_{0}$ and nuclei start dissolving and merging together, the equation of state is not well known. It is possible, that charged pions are produced through $n \rightarrow p+\pi^{-}$, for example, and having spin 0 , then form Bose-Einstein condensates. At the very extreme densities near the centre, we may even get a relativistic Fermi gas of quarks. This picture of a "cold" neutron star is actually valid for interior temperatures up to $10^{10} \mathrm{~K}(1 \mathrm{MeV})$ since, owing to the high densities involved, the crust melting temperature is about $10^{10} \mathrm{~K}$ and the Fermi energies of the quantum liquids are in the $5 \times 10^{11} \mathrm{~K}$ to $5 \times 10^{10} \mathrm{~K}(5$ $50 \mathrm{MeV}$ ) range. Considering that following supernovae explosions, neutron stars are probably born with temperatures of $10^{11} K$, it should take less than a minute to solidify the crust and establish the superfluid interior.

Let us start at this point to examine the cooling of the neutron star. For the purposes of a first order calculation, let us assume a two component thermodyna- 
mic model of a one solar mass neutron star:

1) an isothermal interior starting around the density $\rho \cong 10^{10} \mathrm{~g} / \mathrm{cm}^{3}$, within which almost all of the thermal energy resides:

2) an outer envelope starting with the surface and extending to the isothermal interior along which the temperature increases from $T_{\mathrm{s}}$ of the surface to $T_{\mathrm{c}}$ of the interior (see Shapiro and Teukolsky 1983, Chapter 11 for a review of neutron star cooling).

With such a simple model, the cooling curve of a neutron star would have been similar to that of a soft boiled egg if it were not for the fact that the neutron star interior is at very high temperatures where the dominant cooling is through neutrino emission. As will be estimated below, it takes about $10^{5}$ years for the neutron star to cool down to $T_{\mathrm{c}} \cong 10^{8} \mathrm{~K}$ $\left(T_{\mathrm{s}} \cong 10^{6} \mathrm{~K}\right)$ with neutrino emission, after which the photon emission from the surface becomes the dominant cooling process; i.e., the neutron star starts to cool like the egg. Coming back to the neutrino cooling, it is fortunate that we do not need to worry about the interactions of the neutrinos subsequent to their production; below temperatures of $10^{10} \mathrm{~K}$ the mean free path of the emitted neutrinos is greater than the entire thickness of the neutron star. Hence, we can write down the cooling equation simply as:

$$
d U / d t=C_{v} d T_{c} / d t=-\left(L_{v}+L_{\gamma}\right)
$$

where the rate of change of thermal energy $d U / d t$, which also equals the heat capacity $C_{v}=d U / d T_{c}$ times the rate of temperature change, has been equated to the total energy loss rate, $L_{v}$ due to neutrinos, plus $L_{\gamma}$ due to photons from the surface. The heat capacity of the degenerate fermions (predominantly neutrons) for a one solar mass neutron star, in the absence of superfluidity, can be simply estimated as:

$$
C_{\mathrm{v}} \cong 10^{48}\left(T_{\mathrm{c}} / 10^{9}\right) \mathrm{erg} \mathrm{K}^{-1}
$$

The heat capacity is expected to decrease exponentially as the temperature drops below the transition temperature to the superfluid regime; we ignore the superfluidity effects in our simple estimation of the cooling curve. We can also write down the photon luminosity term, assuming that the emitted radiation is blackbody-like:

$$
L_{\gamma}=4 \pi R^{2} \sigma T_{\mathrm{s}}^{4}
$$

where $R$ is the radius of the neutron star and $\sigma$ is the Stefan-Boltzmann constant.

Let us proceed to examine the neutrino luminosity. In the interior, the interacting constituents are $p, n, e^{-}$. At the temperatures and densities involved, the most important neutrino production pro-

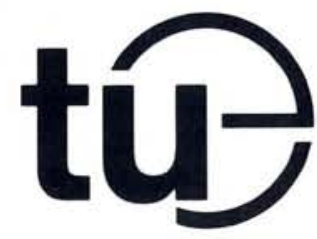

\author{
The Board of the Eindhoven University of Technology, \\ Eindhoven, the Netherlands, \\ announces the vacancy for a
}

\section{FULL PROFESSOR OF PHYSICS $(\mathrm{m} / \mathrm{f})$}

The appointee will work in the group of Applied Nuclear Techniques, part of a division of the Department of Technical Physics that also includes the Plasma, Atomic and Molecular Physics group.

\section{The professor appointed}

- will be expected to carry out and initiate application-oriented research using nuclear physics techniques in interaction with atomic physics, in co-operation with members of the staff and with the second professor in the group, whose main interest is accelerator physics and techniques;

- will have several particle beams (energy up to $40 \mathrm{MeV}$ ) and dye laser beams (an exploratory study of an electron storage ring is in progress) at his disposal; the use of other radiation facilities in and outside the Netherlands through national and international co-operation could be a natural extension of the research programme of the group;

- should have acknowledged qualifications in experimental physics, flexibility and a profound knowledge of the field of research;

- will be expected to be open to interdisciplinary research and to give guidance to the research group with youthful enthusiasm;

- will be expected to obtain financial support from public and industrial funds;

- has to participate in the Department's teaching programme by giving lectures on general physics and by supervising the research work of undergraduate students and of graduate students working on a Ph.D.-thesis:

- should be willing to participate in the administrative activities of the group and of the Department;

- should have an adequate knowledge of the Dutch language within two years after the appointment.

\section{Letters of applications}

- are awaited within six weeks after the date of appearance of this announcement;

- should be accompanied by a detailed curriculum vitae and list of publications as well as the names and addresses of at least two qualified persons who are prepared to give references about the applicant;

- should be addressed, with the indication V7247, to the

Dean of the Department of Technical Physics, Prof. dr. H.M. Gijsman,

Eindhoven University of Technology, Postbus 513,

NL - 5600 MB Eindhoven, the Netherlands.

Additional information may be obtained from the Chairman of the Appointment Committee,

Prof. dr. ir. D.C. Schram, (telephone: (40) 4725 50) or

the Dean of the Department, Prof. dr. H.M. Gijsman (telephone: (40) 472528 ), Eindhoven University of Technology, Postbus 513,

NL - 5600 MB Eindhoven, the Netherlands.

Those wishing to draw the committee's attention to potential candidates are kindly requested to contact the Chairman of the Appointment Committee. 
cess of these particles is the modified URCA process (the name was coined back in 1941 by Gamov and Schönberg after the losers coming in and out of the gambling house of the Casino de Urca in Rio de Janeiro):

$$
\begin{aligned}
& \mathrm{n}+\mathrm{n} \rightarrow \mathrm{n}+\mathrm{p}+\mathrm{e}^{-}+\overline{\mathrm{v}}_{\mathrm{e}} ; \text { and } \\
& \mathrm{n}+\mathrm{p}+\mathrm{e}^{-} \rightarrow \mathrm{n}+\mathrm{n}+\mathrm{v}_{\mathrm{e}}
\end{aligned}
$$

This process is in principle just the neutron decay process: $\mathrm{n} \rightarrow \mathrm{p}+\mathrm{e}^{-}+\overline{\mathrm{v}}_{\mathrm{e}^{\prime}}$ with rearrangement and addition of a bystander neutron in order to conserve momentum in the degenerate environment. The neutrino luminosity from the modified URCA process is estimated to be:

$$
L_{v, \text { URCA }}=5 \times 10^{39}\left(T_{c} / 10^{9}\right)^{8} \mathrm{erg} / \mathrm{s}
$$

Notice the very strong dependence on the temperature which is primarily due to the phase-space factors of the interacting species. Additional processes, such as the nucleon-nucleon bremsstrahlung:

$$
\mathrm{n}+\mathrm{n}(\text { or } \mathrm{p})-\mathrm{n}+\mathrm{n}(\text { or } \mathrm{p})+\mathrm{v}+\overline{\mathrm{v}}
$$

in the interior and electron-nuclei bremsstrahlung:

$$
\mathrm{e}^{-}+(\mathrm{Z}, \mathrm{A}) \rightarrow \mathrm{e}^{-}+(\mathrm{Z}, \mathrm{A})+\mathrm{v}+\overline{\mathrm{v}}
$$

in the crust, are also expected to contribute to a lesser extent to the neutrino luminosity.

So far, we have estimated the heat capacity $C_{v}$ and neutrino luminosity $L_{v}$ as a function of the internal core temperature $T_{c}$, but the surface photon luminosity $L_{\gamma}$ as a function of the surface temperature $T_{\mathrm{s}}$. In order to solve the cooling equation we need to calculate one more relationship, namely that of $T_{\mathrm{c}}$ to $T_{\mathrm{s}}$. Basically, the conductive opacity (thermal insulation) of the envelope determines this relationship, which can be approximated by $T_{\mathrm{c}} \cong 1.6 \times 10^{-3} \mathrm{~T}_{\mathrm{s}}^{9 / 5}$. Plugging this relationship, the estimated internal temperature dependence of the heat capacity, and luminosities back into the cooling equation gives the following general features:

For about $t \cong 10^{5}$ years following its formation, the neutron star cools predominantly by neutrino emission with $T_{\mathrm{c}} \sim$ $t^{-1 / 6}\left(T_{\mathrm{s}} \sim t^{-1 / 11}\right)$. After this time, the photon luminosity becomes the dominant cooling process where $T_{\mathrm{c}} \sim t^{5}\left(T_{\mathrm{s}} \sim\right.$ $\left.t^{-11 / 4}\right)$. At the break point the internal temperature is about $2 \times 10^{8} \mathrm{~K}$, and the surface temperature is about $10^{6} \mathrm{~K}$.

A number of authors have added more thought and physics to the first order calculations discussed above and come up with various alternatives and corrections to the cooling curve (see Tsuruta 1986; and Nomoto and Tsuruta 1987); the range of these differences is summarized in Fig. 2 together with the recent experimental results from EINSTEIN and EXOSAT satellites.

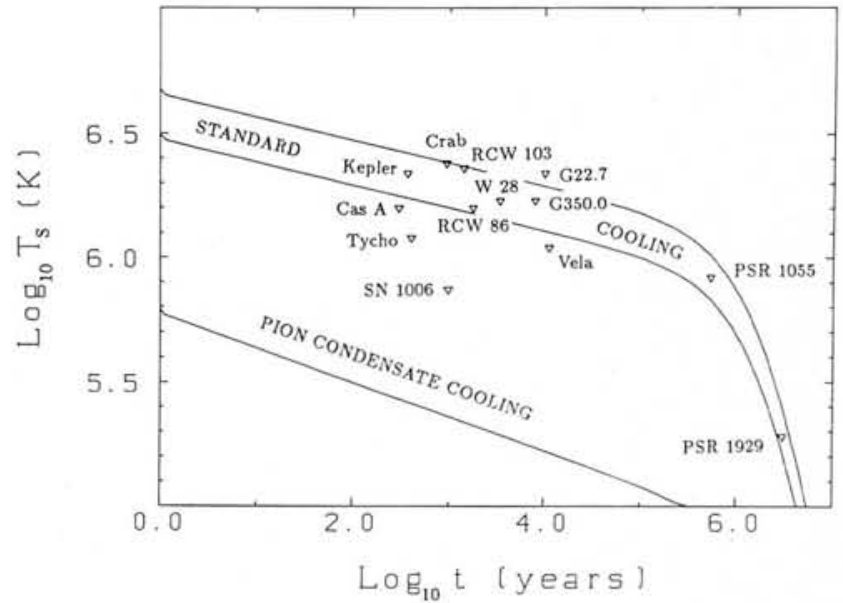

Fig. 2 - Cooling curves for a one solar mass neutron star. The shaded region represents the range of standard cooling calculations with and without superconductivity and finite relaxation time effects; soft and hard equations of state have also been included. The lower curve represents the case of pion condensate cooling. The measured EINSTEIN data points are taken from the summary of Tsuruta (1986), PSR 1929+10 from Alpar et al. (1987), PSR 1055-52 from Brinkmann and Ögelman (1987).

The most dramatic difference to the cooling curve is introduced by the possible existence of pion condensates which increase the neutrino luminosity to:

$$
L_{\mathrm{v}, \pi} \cong 10^{45}\left(T_{\mathrm{c}} / 10^{9}\right)^{6} \mathrm{erg} / \mathrm{s}
$$

As shown in Fig. 2 , in this case the neutron star cools very much faster; typically the interior temperatures should be a factor of 50, and surface temperatures a factor of eight lower than the standard cooling case.

Let us now confront the experiments. How does one go about measuring the cooling curves of neutron stars? By far, the largest fraction of the cooling luminosity comes out as neutrinos. However, the same weak interacting property that lets them come out of the neutron star so easily, makes it very difficult to observe them. So until neutrino detectors improve vastly, we have to rely on the photons from the surface. The surface temperatures of interest lie in the range $3 \times 10^{5} \mathrm{~K}$ to $3 \times 10^{6} \mathrm{~K}$; considering that the neutron star is a small object about $10 \mathrm{~km}$ in size, the total luminosity $L_{\gamma}$ for an object of $T_{\mathrm{s}} \sim 10^{6} \mathrm{~K}$ is of the order of $10^{33} \mathrm{erg} / \mathrm{s}$, about the total luminosity of the Sun. The typical energy of these photons would be in the soft X-ray region, a region which is only accessible for observations above the atmosphere. In this band of the electromagnetic spectrum, the contemporary astronomical instruments employ grazing angle telescopes with imaging instruments at the focus.

The first such satellite payload was the EINSTEIN observatory (1978-1981), followed by the European X-Ray Astronomy Satellite EXOSAT (1983-1986). With the exception of an upper limit of $T_{\mathrm{s}} \lesssim 3 \times 10^{6} \mathrm{~K}$ for the Crab pulsar through lunar occultation experiments, no experimental information on neutron star cooling existed prior to the EINSTEIN - EXOSAT era (for a review of EINSTEIN observatory results see Helfand 1983, and Helfand and Becker 1984; for EXOSAT results see Alpar et al. 1987. and Brinkmann and Ögelman 1987). A major part of the problem stems from the difficulty of finding neutron stars that radiate only their cooling luminosity.

For young neutron stars such as the Crab pulsar (born 1054 AD), where we expect hot conveniently detectable surface conditions, the dynamo action of the fast rotating $(30 \mathrm{~Hz})$ strong magnetic field $\left(10^{12}\right.$ gauss on the surface) creates such large accelerating voltages in the magnetosphere that the ensuing non-thermal high energy radiation, including $\mathrm{X}$-rays, swamps out the radiation due to surface cooling. For the case of a very young neutron star, such as one that may at this moment be lurking inside the supernova 1987A in the Large Magellanic Cloud, there is the additional problem of the ejected outer parts of the progenitor star blocking the emitted radiation. In the case of accreting neutron stars in close binary stellar systems, the surface is reheated to temperatures way above the cooling temperature by the infalling material.

For older pulsars, with ages larger than $10^{4}$ years, compact nebulae of relativistic electrons emitting $X$-rays via synchrotron radiation in the surrounding fields appear to be common (Helfand 1983). Nevertheless, with better angular and spectral resolution of future instruments, we should be able to identify the diffuse shape and the non-thermal spectrum of these compact nebulae. At the present, these measurements are at 
the borderline of instrumental resolution and can easily be mistaken for neutron star cooling.

For pulsars older than $10^{6}$ years, the additional process of reheating, such as that due to internal friction, may also affect the cooling curve. As we have already mentioned, the interior of the neutron star consists of solid and superfluid components. As the pulsar radiates dipole radiation and slows down, the differential rotation rate between the solid and superfluid parts introduces internal friction which eventually is dissipated as heat from the surface. It is estimated that in pulsars with a slow-down rate of $10^{-13} \mathrm{radian} / \mathrm{s}^{2}$, the internal heating should lead to a surface temperature around $2 \times 10^{5} \mathrm{~K}$. From another reheating process, energetic particles reimpacting on the surface, similar temperatures are estimated. So the conclusion is that there may always exist some emission processes in the vicinity of the neutron star which can mask the effect of cooling of the initial heat content. At best, the present measurements should be considered as upper limits.

When we examine the data points in Fig. 2, we can see that most of them lie within the range of standard cooling scenarios. Out of the four objects that fall below this range, only the Vela pulsar is a bona fide neutron star. That is to say, the other three, Cas A, Tycho and SN1006 are historically recorded supernovae, but it is possible that these supernovae have left no neutron star behind. Indeed, Type I supernovae are believed to occur when a white dwarf accretes mass over the Chandrasekhar limit (1.4 $M_{\odot}$ ), and undergoes a thermonuclear explosion. In theoretical studies of these type of explosions, the white dwarf is totally disrupted and no neutron star remains. In the case of the Vela pulsar, however, we not only see a supernova remnant, but we also find a pulsar rotating with a period of 89 milliseconds. Using the size of the supernova remnant or the period and period-derivates of the pulsar, one can extrapolate back and arrive at a consistent age of 11000 years. Being fairly close-by, only 500 parsec $\left(1.6 \times 10^{21} \mathrm{~cm}\right)$ away, both EINSTEIN and EXOSAT observatories have been able to resolve the compact nebula from the central point source that must be the pulsar. One possible way of explaining the low surface temperature of the Vela pulsar, below the standard cooling curves, is to postulate that this neutron star is sufficiently massive $\left(\gtrsim 1.4 M_{\odot}\right)$ and hence of sufficiently high central density that some pion condensate cooling is effective.
In addition to the surface emission, there has been another indirect method proposed to measure the internal temperatures. Alpar et al. (1985) have developed a theory of vortex creep (movement of the quantized rotation of superfluid neutrons through the inner crust lattice) and interpreted the glitches in the rotation rates of $\mathrm{Crab}$ and Vela pulsars. The internal temperature of the neutron star is one parameter in this model which, after theoretical estimates of the other parameters, can be fitted to the data. These measurements give again an internal temperature for $\mathrm{Crab}$ that is consistent with standard cooling. However, for the Vela pulsar this method also gives temperatures lower than that expected from standard cooling.

Summarizing the neutron star cooling story, we can say that at present the measurements are just on the borderline of being accurate enough to restrict or challenge the existing theories and hence models of neutron star interiors. Future satellite programs of the next decade may indeed accomplish this task. Along with experimental improvements, we can expect better theories including the effects of the magnetic fields and other exotic particles. To be able to study hadron physics through observations of neutron star cooling is a fine example of the progress of scientific understanding through solidarity in very diverse fields.

\section{REFERENCES}

Alpar M.A., Nandkumar R. and Pines D., Astrophys. J. 288 (1985) 191.

Alpar A.M., Brinkmann W., Kızıloğlu Ü, Ögelman H. and Pines D., 1987, Astron. Astrophys. 177 (1981) 101.

Brinkmann W. and Ögelman H., Astron. Astrophys. (1987) to be published.

Giacconi R., Gursky H., Paolini F.R. and Rossi B.B., Phys. Rev. Lett. 9 (1962) 439.

Helfand D.J., in Supernova Remnants and their $X$-ray Emission, IAU Symposium No. 101, eds. J. Danziger and P. Gorenstein (Reidel, Dordrecht) 1983, p. 471.

Helfand D.J. and Becker R.H., Nature 307 (1984) 215.

Hewish A., Bell S.J., Pilkington J.D.H., Scott P.F. and Collins R.A., Nature 217 (1968) 709.

Nomoto K. and Tsuruta S., Astrophys. J. 312 (1987) 711.

Pines D., in Proc. of NATO ASI on High Energy Phenomena Around Collapsed Stars, ed. F. Pacini (Reidel, Dordrecht) 1987, p. 193.

Pines D. and Alpar M.A., Nature 316 (1985) 27.

Shapiro S.L. and Teukolsky S.A., in Black holes, white dwarfs, and neutron stars (John Wiley and Sons) 1983.

Tsuruta S., Comments on Astrophysics XI (1986) 151.
SPIE Volume 634

OPTICAL AND HYBRID COMPUTING

\author{
Second in the Series \\ 38 papers, 485 pages \\ ISBN 0-89252-669-6 \\ Copyright 1987 \\ Editor: H. H. Szu, \\ Naval Research Laboratory \\ 24-27 March 1986 \\ Leesburg, Virginia USA
}

In 1986 a small group of about 40 experts came together in a synergistic manner for four days and three nights in a retreat environment and devoted

themselves to assessing and projecting the future of optical computing. This peerreviewed volume is the result of that interaction

Part One

Algorithms for Linear and Nonlinear Systems 13 papers

Part Three

Neural Networks for Computing 8 papers

Part Two

Architectures Based on

Bistable and Molecular Devices 7 papers

Part Four

Application Driven Devices and System Developments Io papers

The concept of the SPIE Institutes for Advanced Optical Technologies developed out of SPIE's desire to foster increased interaction and collaboration among researchers working in emerging optical technologies.

SPIE Member $\$ 65$

Nonmember North America $\$ 79$ Nonmember All Other Countries \$91

To order: contact SPIE, Avenue de la Tanche 2, B-1160 Brussels, Belgium; Telephone 2/660.45.11: Telex 25387 AVVAL B. In North America, contact SPIE, P.O. Box 10, Bellingham, WA 98227.0010 USA:Telephone 206/676-3290; Telex 46-7053. 